\title{
Next-to-leading order QCD corrections to inclusive-hadron photoproduction in polarized lepton-proton collisions
}

\author{
D. de Florian and W. Vogelsang \\ Theory Division, CERN, CH-1211 Geneva 23, Switzerland
}

(Received 8 December 1997; published 5 March 1998)

\begin{abstract}
We calculate the next-to-leading order QCD corrections to the "direct" part of the spin-dependent cross section for single-inclusive charged-hadron photoproduction. This process could be studied experimentally in future polarized fixed-target lepton-nucleon experiments, but also at the DESY ep collider HERA after an upgrade to both beams being polarized. We present a brief numerical evaluation of our results by studying the $K$-factors and the scale dependence of the next-to-leading order cross section. [S0556-2821(98)02209-7]

PACS number(s): 13.88.+e, 12.38.Bx
\end{abstract}

\section{INTRODUCTION}

In the last few years measurements of the spin asymmetries $A_{1}^{N}(N=p, n, d)$ in longitudinally polarized deepinelastic scattering (DIS) have provided much new information on the spin structure of the nucleon. Theoretical leading order (LO) [1-3] and next-to-leading order (NLO) [1-4] analyses of the data sets demonstrate, however, that these are not sufficient to extract accurately the spin-dependent quark $\left(\Delta q=q^{\uparrow}-q^{\downarrow}\right)$ and gluon $\left(\Delta g=g^{\uparrow}-g^{\downarrow}\right)$ densities of the nucleon. This is true in particular for $\Delta g\left(x, Q^{2}\right)$, since it contributes to DIS in LO only via the $Q^{2}$-dependence of $g_{1}$ (or $A_{1}$ ) which could not yet be accurately studied experimentally. As a result of this, it turns out [1-4] that the $x$-shape of $\Delta g$ seems to be hardly constrained at all by the DIS data, even though a tendency towards a fairly large positive total gluon polarization, $\int_{0}^{1} \Delta g\left(x, Q^{2}=4 \mathrm{GeV}^{2}\right) d x \gtrsim 1$, was found $[1,2,4]$. The measurement of $\Delta g$ thus remains one of the most interesting challenges for future spin physics experiments. When selecting suitable processes for a determination of $\Delta g$, it is crucial to pick those that, unlike $g_{1}$, have a gluonic contribution already at the lowest order. Sticking to polarized lepton-nucleon interactions, this implies to consider processes less inclusive than DIS. Among those is the production of a (charged) hadron with large transverse momentum $p_{T}$. To obtain a large number of such hadrons, it is expedient to go to photoproduction, i.e. to the limit when the (circularly polarized) photon which is exchanged between the polarized lepton and the nucleon, is almost on-shell. In this way one avoids the suppression of the cross section by the photon propagator.

As was shown recently [5], a polarized version of the DESY $e p$ collider HERA with $\sqrt{s} \approx 300 \mathrm{GeV}$ would be a very promising and useful facility for studying polarized photoproduction reactions. In particular, two of the conceivable processes, single-inclusive hadron production and jet production, show strong sensitivity to the polarized gluon distribution of the proton and also appear likely to yield statistics good enough for a successful measurement [5]. In the framework of the LO calculation performed in [5], the sensitivity of these reactions to $\Delta g$ is due in the first place to the subprocess $\vec{\gamma} \vec{g} \rightarrow q \bar{q}$, where the arrows denote longitudinal polarization. As was stressed in [5], and as is well- established in the unpolarized case, the (quasi-real) photon will not only interact in a direct ("pointlike") way, but can also be resolved into its hadronic structure. As far as a determination of $\Delta g$ is concerned, such "resolved" contributions (which appear at the same order in perturbation theory as the "direct" piece) are to be considered as a background. As was shown in [5], the resolved component is subdominant with respect to the direct one in certain regions of rapidity and transverse momentum of the produced hadron or jet, thus maintaining the clear-cut sensitivity to $\Delta g$ resulting from the direct piece. Focusing on the other hand on the resolved component, the study of polarized photoproduction at HERA might even allow a measurement of the parton content of polarized photons in the long run [5] - a unique task for HERA which makes the polarization upgrade option of HERA appear even more fascinating.

Polarized photoproduction reactions can also be studied in fixed target experiments with polarized lepton beam and polarized target, like the future COMPASS experiment at CERN, or HERMES at DESY. Among other things, one could look for charged tracks with large $p_{T}$ also in these experiments, whereas the energies would obviously not be large enough for producing decent jets. The resolved component at fixed target energies is expected to be generally negligible.

In order to make reliable quantitative predictions for a high-energy process such as polarized inclusive-hadron photoproduction, it is crucial to extend LO studies such as the one of [5] to NLO by determining the $\mathcal{O}\left(\alpha_{s}\right)$ QCD corrections. The key issue here is to check the perturbative stability of the process considered, i.e. to examine to what extent NLO corrections affect the cross sections and spin asymmetries relevant for experimental measurements. Only when the corrections are reasonably small and under control, can a process that shows good sensitivity to, say, $\Delta g$ at the lowest order, be regarded as a genuine probe of the polarized gluon distribution and be reliably used to extract it from future data. The first basic ingredient for such an extension to NLO has been provided in the past two years by the NLO fits to polarized DIS data mentioned above, which yielded spindependent nucleon parton distributions evolved to NLO accuracy. Focusing on the direct part of inclusive-hadron photoproduction, the calculation of the polarized cross section to 
NLO is then completed by using also (unpolarized) NLO fragmentation functions for the produced hadron (as provided in [6]), and by including the $\mathcal{O}\left(\alpha_{s}\right)$ corrections to the spin-dependent "direct" subprocess cross sections for the inclusive production of a certain parton that fragments into the hadron. The calculation of the latter is the purpose of this paper.

An immediate problem arises here, as the direct part on its own is no longer a really well-defined quantity beyond the LO. This is due to the fact that beyond LO collinear singularities appear in the calculation of the subprocess cross sections for photon-parton scattering which are to be attributed to a collinear splitting of the photon into a $q \bar{q}$ pair and need to be absorbed into the photon structure functions. As the latter only appear in the resolved part of the cross section, and since factorizing singularities is never a unique procedure, it follows that only the sum of the direct and the resolved pieces is independent of the factorization scheme chosen and thus is physical. This has been known for a long time from the unpolarized case where the corrections to the direct $[7,8]$ and to the resolved [9] contributions have all been calculated. Nevertheless, we will concentrate in this work only on the corrections to the direct part of the polarized cross section, mainly because this calculation-albeit already being quite involved-is much simpler than the one for the resolved piece. Our results will therefore only be the first step in a full calculation of NLO effects to polarized inclusive-hadron photoproduction. Despite the fact that they are not complete in the sense discussed above, we believe our results to be very important, both phenomenologically and theoretically: As mentioned earlier, the direct component dominates at fixed target energies and also still for the HERA collider situation in certain regions of phase space. This means that our NLO results should be rather close to the true NLO answer in these cases even if the resolved component is only taken into account on a LO basis, which in turn implies that our NLO corrections should already be sufficient to shed light on the question of general perturbative stability of the process. We also mention in this context that our results for the NLO corrections to the direct hard subprocess cross sections will help to obtain or to check those for the resolved ones as the Abelian ("QED-like") parts of the two are the same.

The paper is organized as follows: in Sec. II we present the calculation of the $\mathcal{O}\left(\alpha_{s}\right)$ corrections to the direct part of polarized inclusive-parton photoproduction. Section III is devoted to a brief numerical evaluation of our results for HERA and fixed target kinematics. Section IV contains the conclusions.

\section{CALCULATION OF THE NLO CORRECTIONS TO THE DIRECT PART OF POLARIZED INCLUSIVE-PARTON PHOTOPRODUCTION}

\section{A. General framework}

The process we want to study is the single-inclusive production of a hadron $h$ in photoproduction in collisions of longitudinally polarized electrons (or muons) and protons, i.e. $\vec{e}\left(p_{e}\right) \vec{p}\left(p_{p}\right) \rightarrow h\left(p_{h}\right) X$. The NLO expression for the corresponding spin-dependent cross section is given by

$$
\begin{aligned}
E_{h} \frac{d \Delta \sigma^{h}}{d^{3} p_{h}} \equiv & \frac{1}{2}\left(E_{h} \frac{d \sigma_{++}^{h}}{d^{3} p_{h}}-E_{h} \frac{d \sigma_{+-}^{h}}{d^{3} p_{h}}\right) \\
= & \frac{1}{\pi S} \sum_{i, j} \int_{1-V+V W}^{1} \frac{d z}{z^{2}} \int_{V W / z}^{1-(1-V) / z} \\
& \times \frac{d v}{v(1-v)} \int_{V W / v z}^{1} \frac{d w}{w} \\
& \times \Delta f_{\gamma}^{e}\left(x_{e}, M^{2}\right) \Delta f_{i}^{p}\left(x_{p}, M^{2}\right) D_{j}^{h}\left(z, M_{F}^{2}\right) \\
& \times \frac{\pi \alpha_{s}\left(\mu^{2}\right) \alpha_{e m}}{s}\left(\frac{d \Delta \hat{\sigma}_{\gamma i \rightarrow j}^{(0)}(v)}{d v} \delta(1-w)\right. \\
& \left.+\frac{\alpha_{s}\left(\mu^{2}\right)}{\pi} \frac{d \Delta \hat{\sigma}_{\gamma i \rightarrow j}^{(1)}}{d v d w}\left(s, v, w, \mu^{2}, M^{2}, M_{F}^{2}\right)\right),
\end{aligned}
$$

the subscripts ",++ , " +- " in Eq. (1) denoting the settings of the helicities of the incoming electron and proton. We have introduced the hadronic variables

$$
\begin{aligned}
& V \equiv 1+\frac{T}{S}, \quad W \equiv \frac{-U}{S+T}, \\
& S \equiv\left(p_{e}+p_{p}\right)^{2}, \quad T \equiv\left(p_{e}-p_{h}\right)^{2}, \quad U \equiv\left(p_{p}-p_{h}\right)^{2},
\end{aligned}
$$

and the partonic ones

$$
\begin{aligned}
& v \equiv 1+\frac{t}{s}, \quad w \equiv \frac{-u}{s+t}, \\
& s \equiv\left(p_{\gamma}+p_{i}\right)^{2}, \quad t \equiv\left(p_{\gamma}-p_{j}\right)^{2}, \quad u \equiv\left(p_{i}-p_{j}\right)^{2} .
\end{aligned}
$$

Neglecting all masses, one has the relations

$$
\begin{aligned}
& s=x_{e} x_{p} S, \quad t=\frac{x_{e}}{z} T, \quad u=\frac{x_{p}}{z} U, \\
& x_{e}=\frac{V W}{v w z}, \quad x_{p}=\frac{1-V}{z(1-v)},
\end{aligned}
$$

where $x_{e}\left(x_{p}\right)$ is the fraction of the longitudinal momentum of the electron (proton) taken by the photon (by parton $i$ ). Similarly, $z$ is the momentum share that hadron $h$ inherits from its parent parton $j$ in the fragmentation process. The spin-dependent ("'helicity-weighted") parton distributions of electrons and protons that appear in the expression (2) for the polarized cross section are defined as usual by

$$
\Delta f_{i}^{e, p}\left(x, M^{2}\right) \equiv f_{i(+)}^{e, p(+)}\left(x, M^{2}\right)-f_{i(+)}^{e, p(-)}\left(x, M^{2}\right),
$$

where $f_{i(+)}^{e, p(+)}\left(x, M^{2}\right)\left(f_{i(+)}^{e, p(-)}\left(x, M^{2}\right)\right)$ denotes the probability at scale $M$ of finding parton $i$ with positive helicity and momentum fraction $x$ in an electron or proton with positive (negative) helicity. As we only deal with the direct case, the only parton type occurring for the polarized electron struc- 
ture functions is the photon, and the $\Delta f_{\gamma}^{e}$ coincide with the spin-dependent Weizsäcker-Williams spectrum ${ }^{1}[10]$ :

$$
\begin{aligned}
\Delta f_{\gamma}^{e}\left(y, M^{2}\right) & \equiv \Delta P_{\gamma / e}(y) \\
& =\frac{\alpha_{e m}}{2 \pi}\left(\frac{1-(1-y)^{2}}{y}\right) \ln \frac{Q_{m a x}^{2}(1-y)}{m_{e}^{2} y^{2}},
\end{aligned}
$$

with $m_{e}$ being the electron (or muon) mass and $Q_{\max }^{2}$ the allowed upper limit on the radiated photon's virtuality, to be fixed by the experimental conditions. The fragmentation function $D_{j}^{h}\left(z, M_{F}^{2}\right)$ in Eq. (2), describing the fragmentation process $j \rightarrow h$, is of course the usual unpolarized one, since we sum over all polarizations in the final state.

Finally, the spin-dependent LO and NLO cross sections for the subprocesses $\gamma i \rightarrow j X, \quad d \Delta \hat{\sigma}_{\gamma i \rightarrow j}^{(0)} / d v$ and $d \Delta \hat{\sigma}_{\gamma i \rightarrow j}^{(1)} / d v d w$, which have been stripped of trivial factors involving the electromagnetic coupling constant $\alpha_{e m}$ and the strong one $\alpha_{s}\left(\mu^{2}\right)$, are defined in complete analogy with Eq. (1). Note that, as indicated in Eq. (2), $d \Delta \hat{\sigma}_{\gamma i \rightarrow j}^{(1)} / d v d w$ will explicitly depend on the renormalization scale $\mu$ as a result of the renormalization procedure for the NLO virtual corrections, and also on the scales $M, M_{F}$ of the parton distributions and fragmentation functions, owing to the factorization of initial and final state collinear singularities. The calculation of the $d \Delta \hat{\sigma}_{\gamma i \rightarrow j}^{(1)} / d v d w$ is the purpose of this paper.

To conclude this section, let us note that the expression for the unpolarized cross section for single-inclusive hadron photoproduction is similar to the one in Eqs. (1) and (2), taking the sum instead of the difference in Eq. (1) and using unpolarized subprocess cross sections $d \hat{\sigma}_{\gamma i \rightarrow j}^{(0)} / d v$, $d \hat{\sigma}_{\gamma i \rightarrow j}^{(1)} / d v d w$ and parton distributions in Eq. (2). The latter correspond to taking the sum instead of the difference in Eq. (6), and for the electron case the unpolarized WeizsäckerWilliams spectrum [10] is obtained from Eq. (7) by replacing $1-(1-y)^{2} \rightarrow 1+(1-y)^{2}$. When calculating the polarized $d \Delta \hat{\sigma}_{y i \rightarrow j}^{(1)} / d v d w$, we will at the same time also determine their unpolarized counterparts and compare them to existing analytical results in the literature [8]. This will serve as a very good check on our calculation. Furthermore, the unpolarized cross section is needed when one wants to calculate spin asymmetries, defined by

$$
A^{h} \equiv \frac{E_{h} d \Delta \sigma^{h} / d^{3} p_{h}}{E_{h} d \sigma^{h} / d^{3} p_{h}}
$$

which are usually the only quantities directly accessible to experiment.

\footnotetext{
${ }^{1}$ For the resolved contribution, one has $\Delta f_{\gamma}^{e} \rightarrow \Delta f_{k}^{e}$ in Eq. (2), where $\Delta f_{k}^{e}$ is a convolution of $\Delta f_{\gamma}^{e}$ in Eq. (7) with the polarized photon structure function for parton type $k$.
}

\section{B. LO contributions}

The subprocesses contributing to $\Delta \hat{\sigma}_{\gamma i \rightarrow j}^{(0)}$ are

$$
\begin{aligned}
& \vec{\gamma} \vec{q} \rightarrow g(q), \\
& \vec{\gamma} \vec{q} \rightarrow q(g), \\
& \vec{\gamma} \vec{g} \rightarrow q(\bar{q}) .
\end{aligned}
$$

Here it is understood that the final-state particle in brackets is unobserved and integrated over its entire phase space, while the other fragments into the hadron. Note that the last process in Eq. (9) is symmetric under exchange of $q, \bar{q}$. The corresponding spin-dependent cross sections read

$$
\begin{aligned}
& \frac{d \Delta \hat{\sigma}_{\gamma q \rightarrow g}^{(0)}(v)}{d v}=2 C_{F} e_{q}^{2} \frac{1-v^{2}}{v}, \\
& \frac{d \Delta \hat{\sigma}_{\gamma q \rightarrow q}^{(0)}(v)}{d v}=2 C_{F} e_{q}^{2} \frac{1-(1-v)^{2}}{1-v}, \\
& \frac{d \Delta \hat{\sigma}_{\gamma g \rightarrow q}^{(0)}(v)}{d v}=-2 T_{R} e_{q}^{2} \frac{v^{2}+(1-v)^{2}}{v(1-v)},
\end{aligned}
$$

where $C_{F}=4 / 3, T_{R}=1 / 2$, and $e_{q}$ is the fractional charge of the quark.

\section{NLO contributions}

Apart from the generic inclusive processes $\gamma q \rightarrow g, \gamma q$ $\rightarrow q$, and $\gamma g \rightarrow q$ that are already present at the LO level, there are also contributions that can arise only beyond the Born approximation. These are $\gamma g \rightarrow g, \gamma q \rightarrow \bar{q}$, and $\gamma q$ $\rightarrow q^{\prime}$, where in the latter process $q^{\prime}$ denotes a quark (or antiquark) of flavor different from $q$. This means that the following explicit subprocesses have to be evaluated:

(a) the interference between the Born graphs $\vec{\gamma} \vec{q} \rightarrow g(q)$, $\vec{\gamma} \vec{q} \rightarrow q(g), \quad \vec{\gamma} \vec{g} \rightarrow q(\bar{q})$ and the virtual corrections to them,

(b) the real corrections to the Born graphs, $\vec{\gamma} \vec{q} \rightarrow g(q g)$, $\vec{\gamma} \vec{g} \rightarrow q(\bar{q} g)$, and

$$
\overrightarrow{\gamma q} \rightarrow q\left\{\begin{array}{l}
(g g) \\
(q \bar{q}) \\
\left(q^{\prime} \bar{q}^{\prime}\right)
\end{array}\right.
$$

(note that for the latter contribution a finite answer is obtained only if all three subprocesses are added),

(c) $\vec{\gamma} \vec{g} \rightarrow g(q \bar{q}), \vec{\gamma} \vec{q} \rightarrow \bar{q}(q q), \vec{\gamma} \vec{q} \rightarrow q^{\prime}\left(q \bar{q}^{\prime}\right)$. 


\section{Regularization of singularities}

It is well known that singularities are encountered when calculating the loop diagrams or when performing the phase space integrations for the unobserved partons in the $2 \rightarrow 3$ processes: first of all, the loop-diagrams contain ultraviolet divergencies which are removed by renormalization. Adding the renormalized loop and the corresponding $2 \rightarrow 3$ contributions, the infrared singularities which are individually present in both parts, also cancel out, and one is left with collinear singularities which are finally removed by the factorization procedure (for the contributions from (c) only singularities of the latter type occur). Of course, for being able to handle the singularities, one has to choose a consistent method of regularization. In our calculation we use dimensional regularization for this purpose, where $d=4-2 \epsilon$, which is the most convenient and customary choice.

The calculation of the spin-dependent squared matrix elements requires projection onto definite helicity states of the incoming particles (which are taken to have momenta $p_{1}$, $\left.p_{2}\right)$, which is achieved by using the relations

$$
u\left(p_{1}, h_{q}\right) \bar{u}\left(p_{1}, h_{q}\right)=\frac{1}{2} p_{1}\left(1-h_{q} \gamma_{5}\right)
$$

for incoming quarks with helicity $h_{q}$ (analogously for antiquarks) and

$$
\begin{aligned}
& \epsilon^{\mu}\left(p_{2}, \lambda_{g}\right) \epsilon^{* \nu}\left(p_{2}, \lambda_{g}\right)=\frac{1}{2(1-\epsilon)}\left(-g^{\mu \nu}+\frac{1}{p_{1} \cdot p_{2}}\left(p_{1}^{\mu} p_{2}^{\nu}\right.\right. \\
& \left.\left.+p_{1}^{\nu} p_{2}^{\mu}\right)\right)+\frac{i \lambda_{g}}{2 p_{1} \cdot p_{2}} \epsilon_{\rho \sigma}^{\mu \nu} p_{2}^{\rho} p_{1}^{\sigma}
\end{aligned}
$$

for incoming gluons with helicity $\lambda_{g}$. The parts independent of $h_{q}$ and $\lambda_{g}$ contribute to the unpolarized matrix elements, for which the averaging of gluon spins in $d$ dimensions should be performed by dividing by the $d-2=2(1-\epsilon)$ possible spin orientations, as has been made explicit in Eq. (12).
As is well known, the use of $\gamma_{5}$ and the Levi-Civita tensor appearing in Eqs. (11) and (12) is not entirely straightforward in $d \neq 4$ dimensions. For our calculations we will use the original prescription of 't Hooft and Veltman [11], afterwards systematized by Breitenlohner and Maison [12] (HVBM scheme), which is usually regarded as the most reliable scheme in the sense that its internal algebraic consistency is well established. In this scheme explicit definitions for $\gamma_{5}$ and $\epsilon_{\mu \nu \rho \sigma}$ are given. In particular, $\gamma_{5}$ $\equiv i \epsilon^{\mu \nu \rho \sigma} \gamma_{\mu} \gamma_{\nu} \gamma_{\rho} \gamma_{\sigma} / 4$ !, the $\epsilon$-tensor being regarded as a genuinely four-dimensional object with its components vanishing in all unphysical dimensions. In this way the $d$-dimensional Minkowski space is explicitly divided into two subspaces, a four-dimensional one and a $(d-4)$-dimensional one, each of them equipped with its metric tensor. As a result, apart from $d$-dimensional scalar products $p \cdot q$ (the usual Mandelstam variables), also their respective "subspace" counterparts can show up in calculations, which renders the calculation of traces and phase space integrations somewhat more complicated. Fortunately, we can rely in our calculation to a certain extent on known results, as will be discussed in the next subsection.

\section{E. Virtual corrections and $2 \rightarrow 3$ matrix elements}

In [13] the NLO corrections to the ("nonfragmentation') part of the hadronic single-spin cross section for the production of circularly polarized prompt photons, i.e. the QCD corrections for $\vec{p} p \rightarrow \vec{\gamma} X$, were calculated. This calculation involved the virtual corrections to the Born graphs $\vec{q} g \rightarrow \vec{\gamma} q, \vec{g} q \rightarrow \vec{\gamma} q, \vec{q} \vec{q} \rightarrow \vec{\gamma} g$, as well as the $2 \rightarrow 3$ matrix elements $\vec{a} b \rightarrow \vec{\gamma} c d$. These ingredients were obtained in [13] in the HVBM scheme. We therefore can get the virtual corrections for $\vec{\gamma} \vec{q} \rightarrow g q, \vec{\gamma} \vec{q} \rightarrow q g, \vec{\gamma} \vec{g} \rightarrow q \bar{q}$ and the 2 $\rightarrow 3$ cross sections $\vec{\gamma} \vec{a} \rightarrow b c d$ by appropriately crossing the polarized photon with the unpolarized incoming parton in the results of [13], which greatly facilitates the calculation.

The virtual corrections obtained in this way read, in the modified minimal subtraction $(\overline{\mathrm{MS}})$ scheme,

$$
\begin{aligned}
\frac{d \Delta \hat{\sigma}_{q \gamma \rightarrow q}^{(1), V}}{d v d w}= & \frac{C_{F} e_{q}^{2} \mu^{2 \epsilon}}{\Gamma(1-2 \epsilon)}\left(\frac{\left(4 \pi \mu^{2}\right)^{2}}{s^{2} v(1-v)}\right) \epsilon(1-w)\left[-\frac{2 C_{F}+N_{C}}{\epsilon^{2}} \delta T_{q \gamma}-\frac{1}{\epsilon}\left(b_{0} \delta T_{q \gamma}-2 C_{F} \ln v_{1} \delta T_{q \gamma}\right.\right. \\
& \left.+N_{C} \delta T_{q \gamma} \ln \frac{v_{1}}{v}+N_{C} \frac{v_{1}^{2}}{v}+C_{F} \frac{v_{1}}{v}(5+v)\right)+b_{0} \ln \frac{\mu^{2}}{s} \delta T_{q \gamma}-\left(2 C_{F}-N_{C}\right) \ln v_{1} \ln v \frac{1-2 v}{v} \\
& +\left(2 C_{F}-N_{C}\right) \ln ^{2} v \frac{1-2 v}{2 v}+C_{F} \ln v \frac{3-2 v}{v}-b_{0} \frac{v_{1}^{2}}{v}+\left(2 C_{F}-N_{C}\right) \frac{v_{1}^{2}}{v} \ln v_{1}-2 C_{F} v_{1} \frac{5+2 v}{v} \\
& \left.+C_{F} \pi^{2} \frac{2-6 v+v^{2}}{3 v}+N_{C} \ln v \frac{1-v+v^{2}}{v}-N_{C} \pi^{2} \frac{1-6 v+2 v^{2}}{6 v}\right]
\end{aligned}
$$

$\frac{d \Delta \hat{\sigma}_{q \gamma \rightarrow g}^{(1), V}}{d v d w}=\frac{d \Delta \hat{\sigma}_{q \gamma \rightarrow q}^{(1), V}}{d v d w}[v \leftrightarrow(1-v)]$, 


$$
\begin{aligned}
\frac{d \Delta \hat{\sigma}_{g \gamma \rightarrow q}^{(1), V}}{d v d w}= & \frac{T_{R} e_{q}^{2} \mu^{2 \epsilon}}{\Gamma(1-2 \epsilon)}\left(\frac{\left(4 \pi \mu^{2}\right)^{2}}{s^{2} v(1-v)}\right)^{\epsilon} \delta(1-w)\left(-\frac{2 C_{F}+N_{C}}{\epsilon^{2}} \delta T_{g \gamma}-\frac{1}{\epsilon}\left(b_{0} \delta T_{g \gamma}+3 C_{F} \delta T_{g \gamma}-N_{C} \delta T_{g \gamma} \ln \left(v v_{1}\right)\right)\right. \\
& +N_{C} \ln \left(v v_{1}\right)-7 C_{F} \delta T_{g \gamma}+b_{0} \ln \frac{\mu^{2}}{s} \delta T_{g \gamma}-N_{C} \delta T_{g \gamma} \ln v_{1} \ln v+\frac{1}{6}\left(4 C_{F}-N_{C}\right) \pi^{2} \delta T_{g \gamma}-C_{F} \ln v \frac{3-v}{v} \\
& \left.-C_{F} \ln v_{1} \frac{2+v}{1-v}-\left(2 C_{F}-N_{C}\right) \ln ^{2} v \frac{1+v^{2}}{2(1-v) v}-\left(2 C_{F}-N_{C}\right) \ln ^{2} v_{1} \frac{2-2 v+v^{2}}{2(1-v) v}\right),
\end{aligned}
$$

where $N_{C}=3, b_{0}=11 N_{C} / 6-n_{f} / 3\left(n_{f}\right.$ being the number of active flavors), and $\mu$ is the renormalization scale. Furthermore,

$$
\begin{aligned}
& \delta T_{q \gamma}=\left(1-v^{2}\right) / v, \\
& \delta T_{g \gamma}=-v_{1} / v-v / v_{1},
\end{aligned}
$$

with $v_{1}=1-v$. Note that the result for $\vec{q} \vec{\gamma} \rightarrow q g$ can also be obtained from the one of [14] for $\vec{q} \vec{g} \rightarrow \gamma q$ after crossing and correct adjustment of color.

The integration of the real $2 \rightarrow 3$ matrix elements over the phase space of the unobserved particles has been discussed in detail in $[15,14]$ and need not be recalled here. The technical complications related to the use of the HVBM scheme discussed above have been solved in [14].

Adding the renormalized virtual and the real contributions, all infrared singularities cancel out. In the next section we briefly recall the factorization procedure which removes the remaining collinear singularities.

\section{F. Factorization}

The factorization procedure based on the factorization theorem [16] has been outlined in, for instance, Refs. $[15,14]$. The mass singularities arise when either an incoming particle collinearly emits another particle (or splits into a pair of collinear particles), or when the "observed" final state particle is collinear to an unobserved one. The singular terms attached to the initial legs are separated off at the factorization scale $M$ and absorbed into the initial-state parton distributions which then obey NLO QCD evolution equations. In particular, if the singularity results from a collinear splitting $\gamma \rightarrow q \bar{q}$, it is absorbed into the "pointlike" part of the photon structure function. Of course there is freedom in choosing the factorization prescription, i.e. in subtracting finite pieces along with the pole terms. As already pointed out in the introduction, this is the reason why a separation of direct and resolved contributions to a photoproduction cross section becomes, strictly speaking, meaningless beyond LO. Final state singularities are factorized at the scale $M_{F}$ into the (NLO) unpolarized fragmentation functions $D_{f}^{h}$.

As an example, let us briefly discuss the factorization of the polarized $\vec{q} \vec{\gamma} \rightarrow g(q g)$ subprocess. This is performed in the easiest way by adding a "counter cross section" [15] which, taking into consideration all possible collinear configurations, has the form

$$
\begin{aligned}
\frac{d \Delta \hat{\sigma}_{q \gamma \rightarrow g}^{(1), F}}{d v d w} \sim & -\frac{\alpha_{s}}{2 \pi}\left[\int_{0}^{1} d x_{1} \Delta H_{q q}\left(x_{1}, M^{2}\right) \frac{d \Delta \hat{\sigma}_{\epsilon}^{q \gamma \rightarrow g q}}{d v}\right. \\
& \times\left(x_{1} s, 1+\frac{t}{s}\right) \delta\left[x_{1}(s+t)+u\right] \\
& +\int_{0}^{1} \frac{d x_{3}}{x_{3}^{2}} H_{g g}\left(x_{3}, M_{F}^{2}\right) \frac{d \Delta \hat{\sigma}_{\epsilon}^{q \gamma \rightarrow g q}}{d v} \\
& \times\left(s, 1+\frac{t}{x_{3} s}\right) \delta\left(s+\frac{1}{x_{3}}(t+u)\right) \\
& +\int_{0}^{1} \frac{d x_{3}}{x_{3}^{2}} H_{g q}\left(x_{3}, M_{F}^{2}\right) \frac{d \Delta \hat{\sigma}_{\epsilon}^{q \gamma \rightarrow q g}}{d v} \\
& \left.\times\left(s, 1+\frac{t}{x_{3} s}\right) \delta\left(s+\frac{1}{x_{3}}(t+u)\right)\right] \\
& -\frac{\alpha_{e m}}{2 \pi}\left[\int_{0}^{1} d x_{2} \Delta H_{q \gamma}\left(x_{2}, M^{2}\right) \frac{d \Delta \hat{\sigma}_{\epsilon}^{q q-g g}}{d v}\right. \\
& \left.\times\left(x_{2} s, 1+\frac{t}{x_{2} s}\right) \delta\left[x_{2}(s+u)+t\right]\right],
\end{aligned}
$$

where the $d \Delta \hat{\sigma}_{\epsilon}^{a b \rightarrow c d}(s, v) / d v$ are the polarized $d$-dimensional $2 \rightarrow 2$ cross sections for the processes $a b$ $\rightarrow c d$, to be found for the HVBM scheme in [14]. Furthermore,

$$
(\Delta) H_{i j}\left(z, M^{2}\right) \equiv-\frac{1}{\hat{\epsilon}}(\Delta) P_{i j}(z)\left(\frac{\mu^{2}}{M^{2}}\right)^{\epsilon}+(\Delta) f_{i j}(z),
$$

where $1 / \hat{\epsilon} \equiv 1 / \epsilon-\gamma_{E}+\ln 4 \pi$, as usual in the $\overline{\mathrm{MS}}$ scheme. In Eq. (19) the $(\Delta) P_{i j}(z)$ denote the unpolarized (polarized) one-loop splitting functions for the transitions $j \rightarrow i$ [17]. The functions $(\Delta) f_{i j}(z)$ represent the freedom in choosing a factorization prescription. In the $\overline{\mathrm{MS}}$ scheme these functions vanish. Note that even in the polarized case only the unpolarized $H_{i j}$ contribute to the factorization of final-state singularities, as we do not consider the production of polarized hadrons.

Before proceeding, we have to mention an important subtlety related to the use of the HVBM prescription for $\gamma_{5}$, which affects the polarized function $\Delta H_{q q}$. It is a wellknown property of the HVBM- $\gamma_{5}$ that it leads to helicity non-conservation at the $q q g$ vertex in $d$ dimensions, expressed by a non-vanishing difference of unpolarized and 
polarized $d$-dimensional LO quark-to-quark splitting functions,

$$
\Delta P_{q q}^{4-2 \epsilon}(x)-P_{q q}^{4-2 \epsilon}(x)=4 C_{F} \epsilon(1-x) .
$$

A disagreeable consequence of this is a non-zero first moment ( $x$-integral) of the non-singlet NLO anomalous dimension for the evolution of polarized non-singlet quark densities, in obvious conflict with the conservation of the flavor non-singlet axial current $[18,19,20]$. At the same time, $(20)$ is responsible for producing a result for the $\mathcal{O}\left(\alpha_{s}\right)$ correction to the Bjørken sum rule [21] which disagrees with the one of [22]. These two effects turn out to be closely related, as they can be simultaneously removed by a factorization scheme transformation $[19,20]$, generated by the term on the righthand-side of Eq. (20). In other words, it is advisable, albeit not mandatory in a purely mathematical sense, to deviate slightly from the $\overline{\mathrm{MS}}$ scheme in the polarized case by choosing (see also [20])

$$
\Delta f_{q q}(z)=-4 C_{F}(1-z)
$$

in Eq. (19). The factorization scheme transformation defined by this equation has also been performed in the calculations of the spin-dependent NLO splitting functions $[19,20]$ and is thus respected by the available sets of spin-dependent NLO parton densities $[1-4]$. The " $\gamma_{5}$-effect" described above has been known to occur in the HVBM scheme for quite some time $[23,24,18,25]$ and is obviously a pure artifact of the regularization prescription chosen. Since furthermore physical requirements such as the conservation of the non-singlet axial vector current serve to remove the effect in a straightforward and obvious way, results of NLO calculations in "spin-physics" (such as the ones of $[19,20]$, or ours) are usually regarded as being "genuinely" in the conventional $\overline{\mathrm{MS}}$ scheme only after this transformation has been carried out. The quantities $\Delta f_{q g}, \Delta f_{g q}$, and $\Delta f_{g g}$ in Eq. (19) will of course be set to zero, as in the usual MS scheme. Needless to say that in the unpolarized case $(\overline{\mathrm{MS}})$ one has $f_{q q}=f_{q g}$ $=f_{g q}=f_{g g}=0$.

Another comment concerns the functions $(\Delta) H_{q \gamma}$ needed for factorizing initial-state collinear singularities from photon-splitting to a $q \bar{q}$ pair. As mentioned above, such singularities are absorbed into the "pointlike" part of the photon structure functions. Studies [26,27,28] of the photon structure beyond LO have revealed that the $\mathrm{MS}$-scheme photonic coefficient functions for the photon's DIS structure functions $F_{2}^{\gamma}, g_{1}^{\gamma}$ exhibit a logarithmically singular behavior at large $x$. Combining at NLO the "pointlike" parts of $F_{2}^{\gamma}, g_{1}^{\gamma}$ with estimates for the "hadronic" component based on vector meson dominance (VMD) arguments, one encounters strongly negative results at large $x$, ruling out the use of intuitive VMD ideas in the $\overline{\mathrm{MS}}$ scheme. Instead, an appropriately adjusted ("fine tuned") non-VMD hadronic NLO input would be required in the $\overline{\mathrm{MS}}$ scheme, substantially differing from the LO one, as the only means of avoiding unwanted and physically not acceptable perturbative instabilities for physical quantities like $F_{2}^{\gamma}, g_{1}^{\gamma}$. In the unpolarized case the so-called DIS $\gamma$ factorization scheme [26] was introduced to avoid such "inconsistencies." Here the idea was to absorb the photonic Wilson coefficient for $F_{2}^{\gamma}$ into the photon's quark densities by a factorization scheme transformation, hereby leaving the "hadronic" part untouched. In [28], this procedure was extended to the polarized case. It was found that after transforming to the DIS ${ }_{\gamma}$ scheme, a pure VMD input can be successfully used for phenomenological analyses going beyond the LO. We will therefore specify the functions $(\Delta) f_{q \gamma}$ to be used to transform to the $\operatorname{DIS}_{\gamma}$ scheme. They read:

$$
\begin{aligned}
f_{q \gamma}(x) & =T_{R}\left[\left[x^{2}+(1-x)^{2}\right]\left(\ln \frac{1-x}{x}-1\right)+6 x(1-x)\right], \\
\Delta f_{q \gamma}(x) & =T_{R}\left[(2 x-1)\left(\ln \frac{1-x}{x}-1\right)+2(1-x)\right],
\end{aligned}
$$

where $T_{R}=1 / 2$. Of course, the choice of factorization scheme cannot affect the result for a physical quantity. In other words, in the unpolarized case, where all contributions can be consistently calculated to NLO, it does not matter eventually whether we use photonic parton densities defined in the DIS ${ }_{\gamma}$ or the $\overline{\mathrm{MS}}$ scheme, as long as we use NLO hard cross sections determined in the same scheme. In the polarized case however, we are not yet able to consistently include the NLO "resolved" contributions, as was pointed out several times before. Therefore, comparing the results for the direct part of the NLO cross section in the $\overline{\mathrm{MS}}$ and the DIS ${ }_{\gamma}$ schemes might indicate the uncertainty resulting from not performing a consistent NLO calculation.

\section{G. Final results}

For all processes the final partonic cross section can be cast into the form

$$
\begin{aligned}
\frac{d \Delta \hat{\sigma}_{\gamma i \rightarrow j}^{(1)}}{d v d w}\left(s, v, w, \mu^{2}, M^{2}, M_{F}^{2}\right)= & {\left[\left(c_{a} \delta(1-w)+c_{b} \frac{1}{(1-w)_{+}}+c_{c}\right) \ln \frac{M^{2}}{s}+\left(c_{\tilde{a}} \delta(1-w)+c_{\tilde{b}} \frac{1}{(1-w)_{+}}+c_{\bar{c}}\right) \ln \frac{M_{F}^{2}}{s}\right.} \\
& +\tilde{c}_{1} \delta(1-w) \ln \frac{\mu^{2}}{s}+c_{1} \delta(1-w)+c_{2} \frac{1}{(1-w)_{+}}+c_{3}\left(\frac{\ln (1-w)}{1-w}\right)_{+}+c_{4} \ln v \\
& +c_{5} \ln (1-v)+c_{6} \ln w+c_{7} \frac{\ln w}{1-w}+c_{8} \ln (1-w)+c_{9} \ln (1-v w) \\
& \left.+c_{10} \frac{\ln [(1-v) /(1-v w)]}{1-w}+c_{11} \ln (1-v+v w)+c_{12} \frac{\ln (1-v+v w)}{1-w}+c_{13}\right]
\end{aligned}
$$


Distributions in $w$ such as $\delta(1-w), 1 /(1-w)_{+}$, etc. only occur for the subprocesses that are already present at the Born level. An expression similar to Eq. (23) holds for the unpolarized case with, obviously, different coefficients $c_{i}(v, w)$. We note that we have compared our unpolarized results to the ones presented in an analytical form in [8]. We found an almost complete overall agreement; however, there are a very small number of differences, some of which could be related to typographical mistakes. The only major discrepancies arise for the subprocesses $\gamma q \rightarrow q\left(q^{\prime} \bar{q}^{\prime}\right)$ and $\gamma q$ $\rightarrow q^{\prime}\left(q \bar{q}^{\prime}\right)$. For the first, we believe that the result in [8] was accidentally presented in terms of the "crossed" process $q \gamma \rightarrow q\left(q^{\prime} \bar{q}^{\prime}\right)$. For $\gamma q \rightarrow q^{\prime}\left(q \bar{q}^{\prime}\right)$, it seems that the result in [8] rather corresponds to $\gamma q \rightarrow \bar{q}^{\prime}\left(q q^{\prime}\right)$. Anyway, none of these small discrepancies turns out to have a significant numerical effect. The coefficients $c_{i}(v, w)$ for the unpolarized and polarized cases are rather lengthy and will not be given here. $^{2}$

\section{NUMERICAL RESULTS}

Let us now present some first numerical results for the NLO corrections to polarized single-inclusive photoproduction of charged hadrons. Rather than performing a detailed numerical study of the process, we will restrict ourselves to the most interesting questions. These concern the general size of the corrections (" $K$-factors") and the residual dependence of the NLO cross section on the unphysical scales present in the calculation.

Before starting, we mention that whenever we will calculate the unpolarized NLO cross section, we will do so in a completely consistent way, i.e. by including both the direct and the resolved parts at NLO. Here we make use of our own results for the NLO corrections to the direct part of the cross section (see Sec. II), and of the ones in [9] for the NLO resolved part. Furthermore, we will for consistency use NLO parton densities for the incoming proton [29] and the photon [26], as well as NLO fragmentation functions. For the latter we will use the ones of [6] set up for the sum of charged pions and kaons. They will also be our choice when calculating the polarized cross section.

In the polarized case at NLO, we will use spin-dependent parton distributions for the proton evolved at NLO and fitted to the available DIS data. Several sets for these are available $[1,2,3]$; for definiteness we will choose the ones of [1] determined within the "radiative parton model." These also have the agreeable property of providing parametrizations at NLO and LO, the latter to be used for Born level predictions. In particular, we will choose the "valence" set of [1], which corresponds to the best-fit result of that paper, along with one other set of [1] based on assuming $\Delta g\left(x, \mu^{2}\right)=g\left(x, \mu^{2}\right)$ at the low input scale $\mu$ of [1], where $g\left(x, \mu^{2}\right)$ is the unpolarized Glück-Reya-Vogt (GRV) [29] input gluon distribution. This set will be referred to as "max. gluon" in what follows. Employing these two sets, which both provide a good fit to the available DIS data, but differ significantly in the polar-

\footnotetext{
${ }^{2}$ They can be obtained in a FORTRAN code via electronic mail from Werner.Vogelsang@cern.ch
}

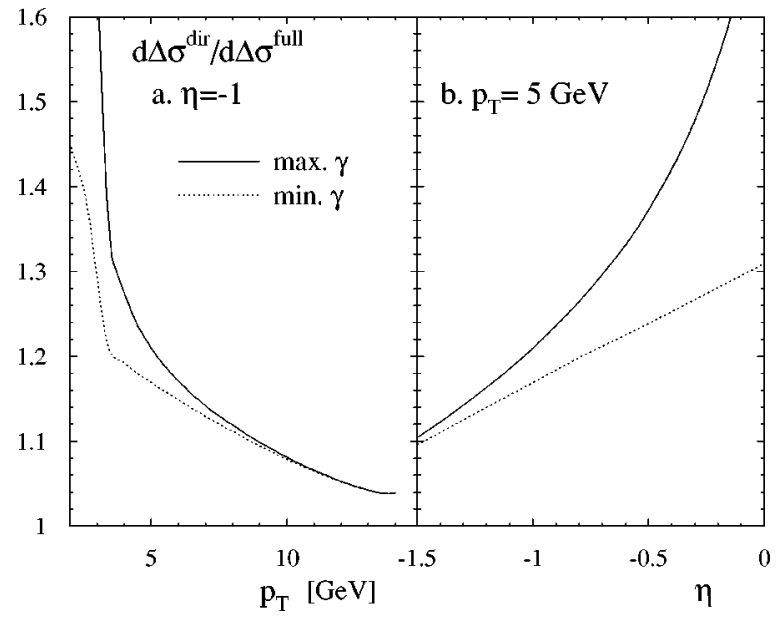

FIG. 1. Ratio of LO direct and full (direct + resolved) polarized cross sections for HERA energies $\left(E_{e}=27 \mathrm{GeV}, E_{p}=820 \mathrm{GeV}\right)$. (a) $p_{T}$ dependence at $\eta=-1$, (b) $\eta$ dependence at $p_{T}=5 \mathrm{GeV}$.

ized gluon density, we are able to see to which extent the relative size of the NLO corrections depends on the set of parton distributions used.

We also note that whenever we calculate a cross section at LO (for instance, when determining the $K$-factor $K$ $=\sigma^{N L O} / \sigma^{L O}$ ), we will for consistency use LO parton distributions and fragmentation functions. In this case we will also use the one-loop expression for the strong coupling, whereas at NLO we obviously employ its two-loop counterpart. The LO and NLO values for the QCD scale parameter $\Lambda_{Q C D}^{\left(n_{f}\right)}$ for $n_{f}$ active flavors are taken from [29,26,1]. Heavy flavor $(c, b)$ contributions to the cross sections are neglected for simplicity. Unless we explicitly study the scale dependence of our results, we will choose the renormalization and factorization scales to be equal to the transverse momentum $p_{T}$ of the produced hadron.

We will provide numerical results for both the fixed target and the HERA collider kinematic domains. While the resolved component is expected to be generally small at fixed target energies, it is known [5] to be dominant in certain regions of phase space at HERA also for the polarized case. Here the direct contribution will dominate only at fairly large $p_{T}$, and/or at negative rapidities $\eta$ of the produced hadron in the HERA laboratory frame, where we have, as usual, counted positive rapidity in the proton forward direction. In order to demonstrate this, and to isolate for our further HERA studies the region where the direct contribution dominates, Fig. 1 shows the ratio of the direct part of the polarized cross section over the full (direct+resolved) one, calculated at LO and plotted vs $p_{T}$ (at $\left.\eta=-1\right)$ and $\eta$ (at $p_{T}$ $=5 \mathrm{GeV})$. We have assumed $E_{e}=27 \mathrm{GeV}$ and $E_{p}$ $=820 \mathrm{GeV}$; the cuts on the polarized Weizsäcker-Williams spectrum were chosen as in [5]. We have used the GlückReya-Stratmann-Vogelsang (GRSV) "max. gluon" set for the polarized proton. For the LO resolved part in the denominator we have to pick a suitable set of LO parton distributions for the polarized photon. Of course, nothing is known as yet experimentally about the latter, so we need to resort to models for them. Here we will follow [5] to use two very different scenarios, first considered in [30]. They are based on assuming "maximal" $\left(\Delta f^{\gamma}\left(x, \mu^{2}\right)=f^{\gamma}\left(x, \mu^{2}\right)\right)$ or "'mini- 


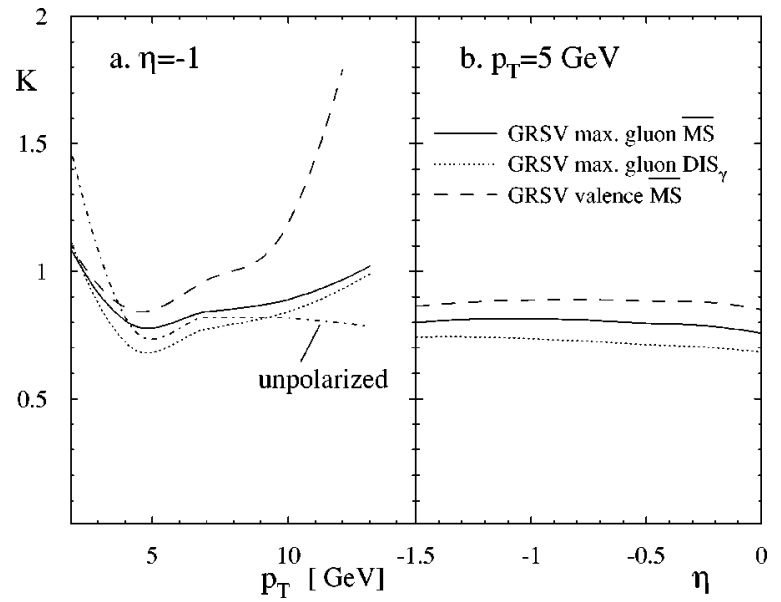

FIG. 2. $K$-factors for the direct part of the polarized cross section at HERA energies for different GRSV [1] parton distributions. In (a) the $\mathrm{K}$-factor for the total unpolarized cross section is also shown.

mal" $\left(\Delta f^{\gamma}\left(x, \mu^{2}\right)=0\right)$ saturation of the fundamental positivity constraints $\left|\Delta f^{\gamma}\left(x, \mu^{2}\right)\right| \leqslant f^{\gamma}\left(x, \mu^{2}\right)$ at the input scale $\mu$ for the QCD evolution, where $\mu$ and the unpolarized photon structure functions $f^{\gamma}\left(x, \mu^{2}\right)$ were adopted from the phenomenologically successful radiative parton model predictions in [26]. These sets will be dubbed "max. $\gamma$ " and "min. $\gamma$ " sets, respectively, and Fig. 1 shows the results obtained for both sets. As can be seen, in the region defined by $\eta \leqslant-1$, $p_{T} \geqslant 5 \mathrm{GeV}$ the resolved component is expected to contribute about $20 \%$ or less to the cross section (note that the direct and resolved parts of the cross section turn out to be of opposite sign).

Having determined the region where the direct component dominates for HERA energies, we can now turn to NLO. Figure 2 shows the $K$-factors for the direct part of the polarized cross section in the $\overline{\mathrm{MS}}$ scheme, again vs $p_{T}$ (at $\eta$ $=-1)$ and $\eta\left(\right.$ at $\left.p_{T}=5 \mathrm{GeV}\right)$. The solid line corresponds to the "max. gluon" set for the polarized parton densities of the proton, whereas the dashed one displays the result obtained within the "valence" best-fit scenario of [1]. As one can clearly see, the $K$-factors are of very moderate size, $K$ $\lesssim 1$ for almost all $p_{T}$ and $\eta$ examined. Only at very large $p_{T}$, near the edge of phase space for the $\eta=-1$ considered, does the $K$-factor become much larger than unity within the "valence" scenario. This finding of generally small NLO corrections is very important and corroborates the LO predictions previously made in [5].

As frequently mentioned earlier, the NLO direct part on its own is factorization scheme dependent. For comparison we also plot in Fig. 2 the $K$-factor for the direct cross section obtained within the DIS ${ }_{\gamma}$ scheme introduced in Sec. II F. As can be seen, the corresponding change of the result is rather small. Finally, Fig. 2 also presents the $K$-factor for the full (direct + resolved) unpolarized cross section, which of course is scheme-independent. It turns out that it is very similar in size and shape to the $K$-factors we have obtained for the direct part of the polarized cross section. This, again, is a very satisfactory finding, as it suggests that our $K$-factor for the direct part might not be too far off the result for the one of the full polarized cross section, to be eventually deter-

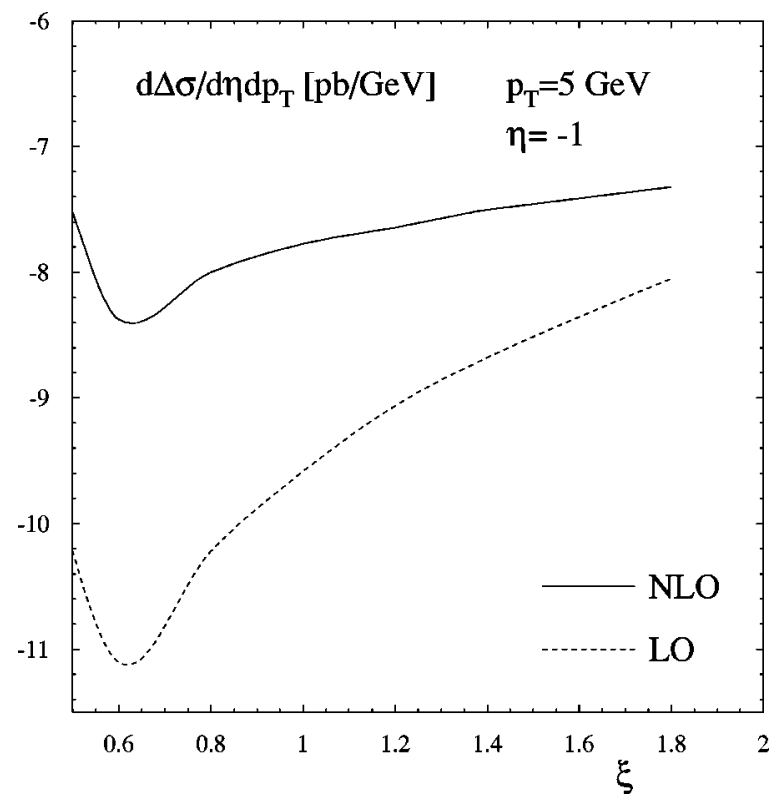

FIG. 3. Scale dependence of the direct part of the polarized inclusive-hadron photoproduction cross section at $p_{T}=5 \mathrm{GeV}$ and $\eta=-1$ for HERA energies. All scales have been set equal to $\sqrt{\xi} p_{T}$, and the parton distributions used correspond to the "max. gluon", set of [1]. The NLO cross section has been calculated in the $\overline{\mathrm{MS}}$ scheme.

mined when the NLO corrections to the resolved part of the polarized cross section will have been calculated.

Another important issue when going beyond the $\mathrm{LO}$ is the expected reduction in the dependence of the results on the unphysical scales $\mu, M, M_{F}$ introduced previously. We now set $\mu^{2}=M^{2}=M_{F}^{2}=\xi p_{T}^{2}$ and plot in Fig. 3 the LO and NLO direct cross sections as functions of $\xi$ for fixed $\eta=-1, p_{T}$ $=5 \mathrm{GeV}$. Even though we can only consider the direct part, the improvement in the scale dependence when going from

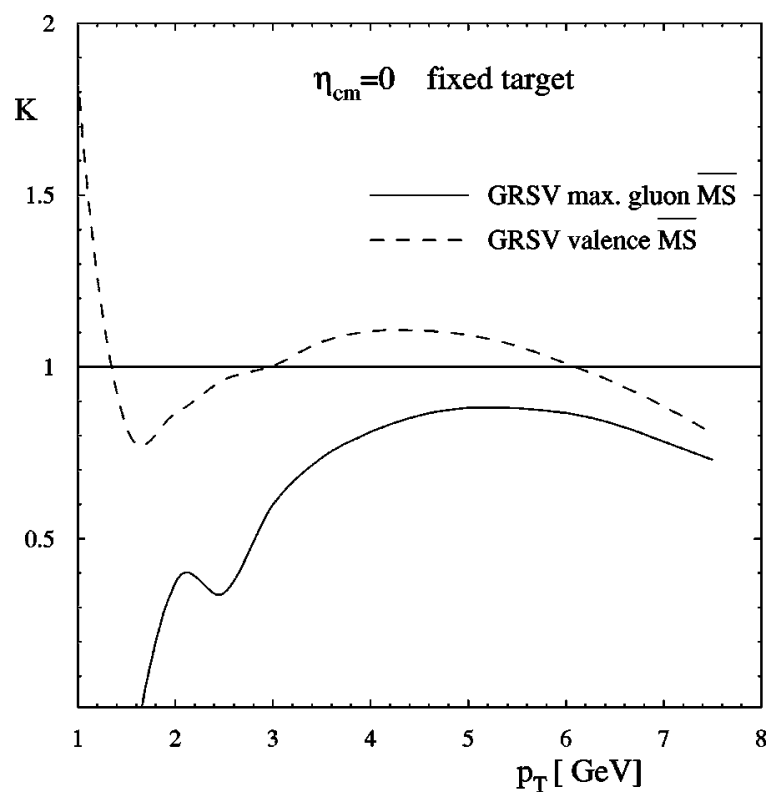

FIG. 4. $K$-factors for the direct part of the polarized singleinclusive charged-hadron cross section in a fixed target experiment with $s=400 \mathrm{GeV}^{2}$ at $\eta_{c m}=0$. 
LO to NLO becomes already clearly visible.

We finally turn to the fixed target region, relevant for the HERMES and the future COMPASS experiments. It is again interesting to study the size of the $K$-factor for this situation, choosing a muon beam energy of $200 \mathrm{GeV}$. The results for our two sets of polarized parton densities of the proton are displayed as functions of $p_{T}$ in Fig. 4, where we have fixed the center-of-mass rapidity, $\eta_{c m}=0$. We have again calculated the NLO cross section in the $\overline{\mathrm{MS}}$ scheme. One can clearly see that again the $K$-factors are of very reasonable size, once $p_{T} \geqslant 3 \mathrm{GeV}$, where one intuitively would start to trust perturbation theory.

\section{SUMMARY AND CONCLUSIONS}

We have presented for the first time the next-to-leading order QCD corrections to the spin-dependent cross section for single-inclusive charged-hadron photoproduction. This process derives its importance from its sensitivity to the proton's spin-dependent gluon distribution and, at high energies, to the so far completely unknown parton content of circularly polarized quasi-real photons. It could be studied experimentally in future polarized fixed-target lepton-nucleon experiments, but also at the DESY ep collider HERA after an upgrade to both beams being polarized.

Our calculation is an important first step in trying to assess the perturbative stability of this process. First numerical results show generally moderate NLO corrections for the direct part of the cross section, the $K$-factor being close to unity over a wide kinematical range at both HERA and fixed target energies. Also, the expected reduction in scale dependence of the cross section when going from LO to NLO is found. We finally emphasize, however, that in order to be able to use our results for obtaining truly physical predictions, the NLO corrections to the resolved part of the cross section will also have to be calculated in the future.

\section{ACKNOWLEDGMENT}

The work of one of us (D.d.F.) was partially supported by the World Laboratory.
[1] M. Glück, E. Reya, M. Stratmann, and W. Vogelsang, Phys. Rev. D 53, 4775 (1996); M. Stratmann, talk presented at the "2nd Topical Workshop on Deep Inelastic Scattering off Polarized Targets: Theory Meets Experiment (SPIN 97)," DESY-Zeuthen, Germany, 1997, Report No. DO-TH 97/22, hep-ph/9710379.

[2] T. Gehrmann and W. J. Stirling, Phys. Rev. D 53, 6100 (1996).

[3] D. de Florian, O. A. Sampayo, and R. Sassot, Phys. Rev. D (to be published), hep-ph/9711440; E. Leader, A. V. Sidorov, and D. B. Stamenov, hep-ph/9708335.

[4] R. D. Ball, S. Forte, and G. Ridolfi, Phys. Lett. B 378, 255 (1996); G. Altarelli, R. D. Ball, S. Forte, and G. Ridolfi, Nucl. Phys. B496, 337 (1997).

[5] M. Stratmann and W. Vogelsang, Z. Phys. C 74, 641 (1997); in Future Physics at HERA, Proceedings of the Workshop, Hamburg, Germany, 1995, edited by G. Ingelman, A. De Roeck, and R. Klanner (DESY, Hamburg, 1996), p. 815; J. M. Butterworth, N. Goodman, M. Stratmann, and W. Vogelsang, Proceedings of the 1997 workshop on "Physics with Polarized Protons at HERA,' Hamburg, Germany, Report No. CERNTH/97-310, hep-ph/9711250.

[6] J. Binnewies, B. A. Kniehl, and G. Kramer, Phys. Rev. D 52, 4947 (1995).

[7] P. Aurenche, A. Douiri, R. Baier, M. Fontannaz, and D. Schiff, Phys. Lett. 135B, 164 (1984); Nucl. Phys. B286, 553 (1987).

[8] L. E. Gordon, Phys. Rev. D 50, 6753 (1994).

[9] F. Aversa, P. Chiappetta, M. Greco, and J. Ph. Guillet, Phys. Lett. B 210, 225 (1988); 211, 465 (1988); Nucl. Phys. B327, 105 (1989).

[10] C. F. von Weizsäcker, Z. Phys. 88, 612 (1934); E. J. Williams, Phys. Rev. 45, 729 (1934).

[11] G. 't Hooft and M. Veltman, Nucl. Phys. B44, 189 (1972).

[12] P. Breitenlohner and D. Maison, Commun. Math. Phys. 52, 11 (1977).
[13] W. Vogelsang, Phys. Rev. D 50, 4436 (1994).

[14] L. E. Gordon and W. Vogelsang, Phys. Rev. D 48, 3136 (1993).

[15] R. K. Ellis, M. A. Furman, H. E. Haber, and I. Hinchliffe, Nucl. Phys. B173, 397 (1980).

[16] D. Amati, R. Petronzio, and G. Veneziano, Nucl. Phys. B146, 29 (1978); A. H. Mueller, Phys. Rev. D 18, 3705 (1978); S. Libby and G. Sterman, ibid. 18, 3252 (1978); R. K. Ellis, H. Georgi, M. Machacek, H. D. Politzer, and G. G. Ross, Nucl. Phys. B152, 285 (1979).

[17] G. Altarelli and G. Parisi, Nucl. Phys. B126, 298 (1977).

[18] M. Stratmann, W. Vogelsang, and A. Weber, Phys. Rev. D 53, 138 (1996).

[19] R. Mertig and W. L. van Neerven, Z. Phys. C 70, 637 (1996).

[20] W. Vogelsang, Phys. Rev. D 54, 2023 (1996); Nucl. Phys. B475, 47 (1996).

[21] J. D. Bjørken, Phys. Rev. 148, 1467 (1966).

[22] J. Kodaira, S. Matsuda, T. Muta, K. Sasaki, and T. Uematsu, Phys. Rev. D 20, 627 (1979); J. Kodaira, S. Matsuda, K. Sasaki, and T. Uematsu, Nucl. Phys. B159, 99 (1979); P. Ratcliffe, ibid. B223, 45 (1983).

[23] A. Weber, Nucl. Phys. B382, 63 (1992).

[24] D. de Florian and R. Sassot, Phys. Rev. D 51, 6052 (1995).

[25] B. Kamal, Phys. Rev. D 53, 1142 (1996); hep-ph/9710374.

[26] M. Glück, E. Reya, and A. Vogt, Phys. Rev. D 45, 3986 (1992); 46, 1973 (1992).

[27] P. Aurenche, M. Fontannaz, and J. Ph. Guillet, Z. Phys. C 64, 621 (1994).

[28] M. Stratmann and W. Vogelsang, Phys. Lett. B 386, 370 (1996).

[29] M. Glück, E. Reya, and A. Vogt, Z. Phys. C 67, 433 (1995).

[30] M. Glück and W. Vogelsang, Z. Phys. C 55, 353 (1992); 57, 309 (1993); M. Glück, M. Stratmann, and W. Vogelsang, Phys. Lett. B 337, 373 (1994). 